Check for updates

Cite this: RSC Adv., 2019, 9, 9401

\title{
Fabrication of gradient vapor grown carbon fiber based polyurethane foam for shape memory driven microwave shielding $\dagger$
}

\begin{abstract}
Yongjie Yan, ${ }^{a}$ Hong Xia, ${ }^{b}$ Yiping Qiu, ${ }^{c}$ Zhenzhen $\mathrm{Xu}^{\mathrm{d}}$ and Qing-Qing Ni (D) *bd
Gradient vapor grown carbon fiber (VGCF) based shape memory polyurethane foam (VGCFaSMPUF) was fabricated by alternate dipping in a gradually diluted VGCF@SMPU/DMF solution and distilled water for shape memory driven microwave shielding. Shape memory performance for this VGCFaSMPUF was achieved by heat transfer of thermally conductive VGCF. Shielding effectivenesS (SE) was adjusted through different degrees of angle recovery. A consistent shielding effect from either side indicated that electromagnetic reflection may take place at both the surface and inside of the non-homogeneous composite shield. For shape memory effect, hot compression made this VGCFCSMPUF achieve a faster recovery time and higher recovery ratio owing to improved thermal conductivity. Moreover, VGCFASMPUF, which was bent to the positive side (PS) with a higher VGCF content, showed shorter recovery time and higher recovery ratio than that bent to the negative side (NS) with a lower VGCF content. We attribute this result to the relatively small mechanical compression strength of the negative side with the lower VGCF content at the bending point when expanding from the positive side. Furthermore, hot compression obviously improved the shielding effectiveness of the VGCFaSMPUF, mainly through a considerable increase of the electrical conductivity. The VGCFASMPUF hot compressed to a thickness of $0.11 \mathrm{~mm}$ achieved a SE value of $\sim 30 \mathrm{~dB}$, corresponding to a shielding efficiency of $\sim 99.9 \%$.
\end{abstract}

Received 2nd January 2019

Accepted 6th March 2019

DOI: $10.1039 / \mathrm{c} 9 \mathrm{ra00028c}$

rsc.li/rsc-advances textiles. Different applications benefit from additional properties such as being light weight and resistant to environmental corrosion oxidation and having high strength and flexibility. ${ }^{\mathbf{1 2 - 1 6}}$ Consequently, carbon/polymer composites, as an important group of carbon composites, are considered important in the field of EM shielding because of the rich variety of available natural and synthetic polymers, which can be modified and adjusted for targeted properties.

Rapid development of advanced intelligence security and smart electronic equipment has led to demand for functional EM shielding devices, which can respond to external stimulus (i.e., electricity, heat, and light) or/and have tunable shielding effect, such as controllable EM shielding switch for cutoff and reception of EM signals. ${ }^{\mathbf{1 7 - 2 4}}$ For example, Wang and co-workers have reported a kind of hydro-sensitive sandwich structure by embedding porous polypropylene non-woven spacers into highly conductive pyrolytic graphite papers. In the presence and absence of polar water molecules, porous spacers achieved a switchable structural transformation through polar induced interfaces. Thus, under dry and wet conditions, the sandwich structures exhibited weak and strong EM shielding ability, respectively. ${ }^{25}$ Furthermore, Chen et al. prepared a biomassderived electrically conductive macroscopic carbon grid (MCG) by carbonizing wood-pulp fabric matting. They found that double-layered MCG showed tunable EM shielding performance by varying the interleaving degree of the stacked 


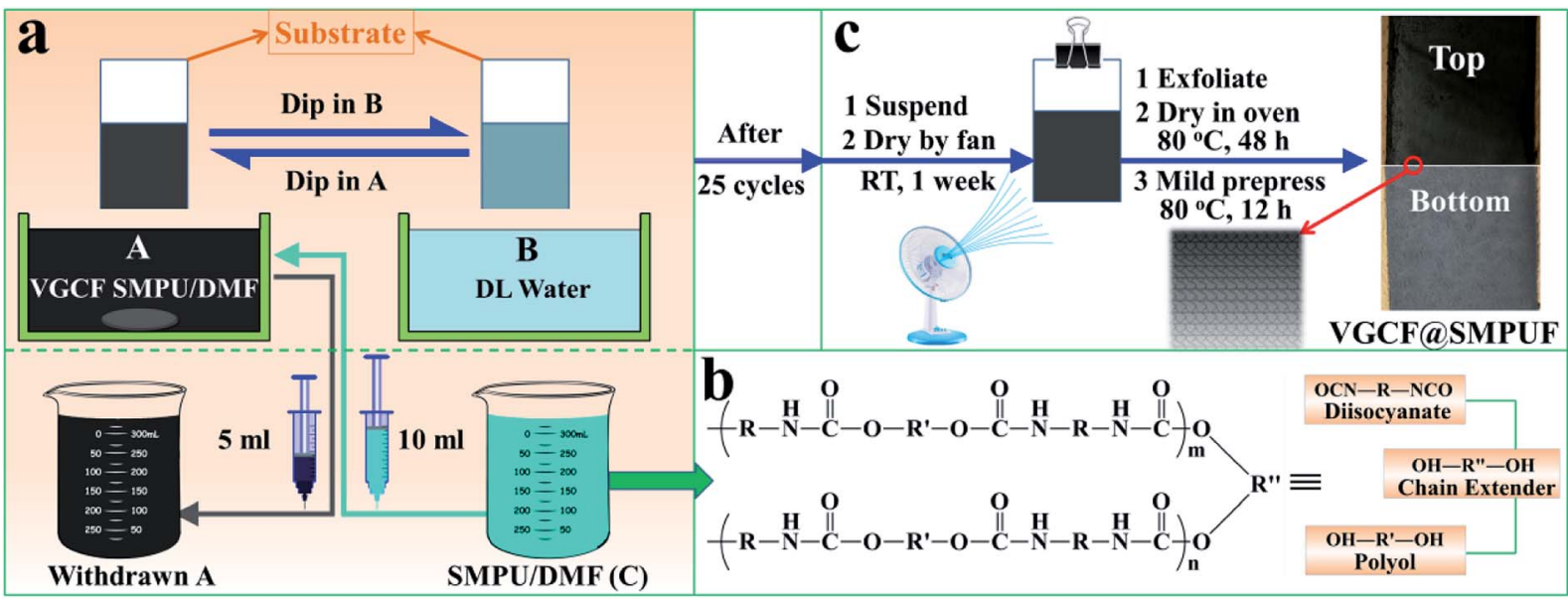

Fig. 1 Schematic illustration showing fabrication of gradient VGCFaSMPUF. (a) Alternative dipping of PP substrate in VGCF(aSMPU/DMF (A) and $D L$ water (B) with partial removal of solution $A$ and addition of solution $C$ after each dipping procedure into solution $A$; (b) chemical structure of SMPU; (c) two-stage drying and hot prepress of VGCF@SMPUF.

grids through tiny translational motion. ${ }^{26}$ Therefore, increasing attention is being paid to developing carbon based shielding composites for functional EM shielding.

In recent years, many types of shape memory polymers (SMPs) containing films, foams, fibers, hydrogels, and fabrics, have been reported, which can respond to various external stimuli such as heat, electricity, light, chemical solvent and $\mathrm{pH}^{.7-33}$ In most cases, shape recovery and fixity effects, which include recovery time, ratio, force, and fixity ratio, were mainly studied to estimate primary performance of those SMPs. However, in terms of the evaluation for their practical applications, little relevant work has been performed except for limited applications, such as soft actuators, functional textures, and medical applications. ${ }^{34-40}$ As for the reason, there is still a limited understanding of how SMPs might be applicable to different fields. Thus, with the aim of expanding applications of SMPs. We therefore introduced shape memory polymers to EM shielding field for the development of functional shielding devices.

In this research, we fabricated gradient vapor grown carbon fiber (VGCF) based shape memory polyurethane foam (VGCF@SMPUF) via alternatively dipping in distilled (DL) water, and a VGCF containing SMPU/DMF solution with a continuously decreasing VGCF concentration, for shape memory driven microwave shielding. The shape memory effect was achieved by heat transfer of thermally conductive VGCF. The shielding effectiveness (SE) of this VGCF@SMPUF was controlled by different degrees of shape recovery. Moreover, the gradient distribution of the VGCF could directionally improve the efficiency of the shape recovery. Furthermore, microwave shielding of this VGCF@SMPUF was also be adjusted by different degrees of hot compression.

\section{Experimental}

\subsection{Materials}

Polyurethane (MM 6520, SMP Technologies Inc, Japan), Vapor grown carbon fiber (VGCF, Showa Denko K. K., Japan), $N, N_{-}$ dimethyl formamide (DMF, Wako Pure Chemical Industries, Ltd), DL water (Wako Pure Chemical Industries, Ltd), and all other reagents were used as received without further purification.

\subsection{Fabrication of gradient VGCF@SMPUF}

On the basis of $\mathrm{H}_{2} \mathrm{O}-\mathrm{DMF}$ solvent exchange, gradient VGCF@SMPUF was fabricated by alternate dipping of a PP substrate in DL water, and VGCF containing SMPU/DMF solution, where the VGCF concentration decreased for later dipping cycles, as illustrated in Fig. 1. Specifically, $2 \mathrm{~g}$ of VGCF (electrical conductivity of $1 \times 10^{4} \mathrm{~S} \mathrm{~cm}^{-1}$, fiber diameter of $\sim 150 \mathrm{~nm}$ ) was at first dispersed in $100 \mathrm{~mL} 80 \mathrm{mg} \mathrm{mL}{ }^{-1}$ of a SMPU/DMF solution by sonication for $1 \mathrm{~h}$ and magnetic stirring for $1 \mathrm{~h}$ in turn. Then, a polypropylene (PP) substrate was alternately dipped into solution A (VGCF@SMPU/DMF), and solvent B (DL water) for 25 cycles. After each dip into solution A, $5 \mathrm{~mL}$ of

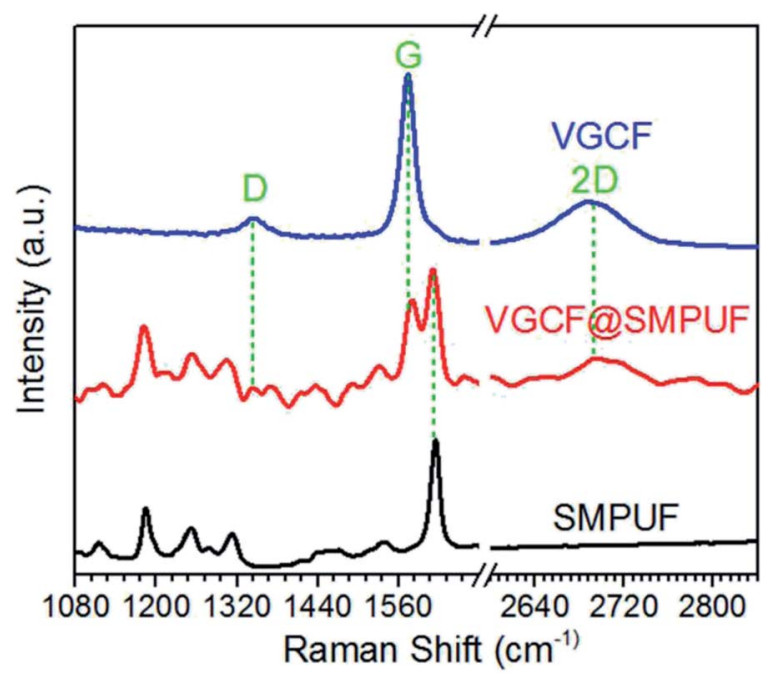

Fig. 2 Raman spectrums of VGCF, SMPUF, and VGCF@SMPUF. 


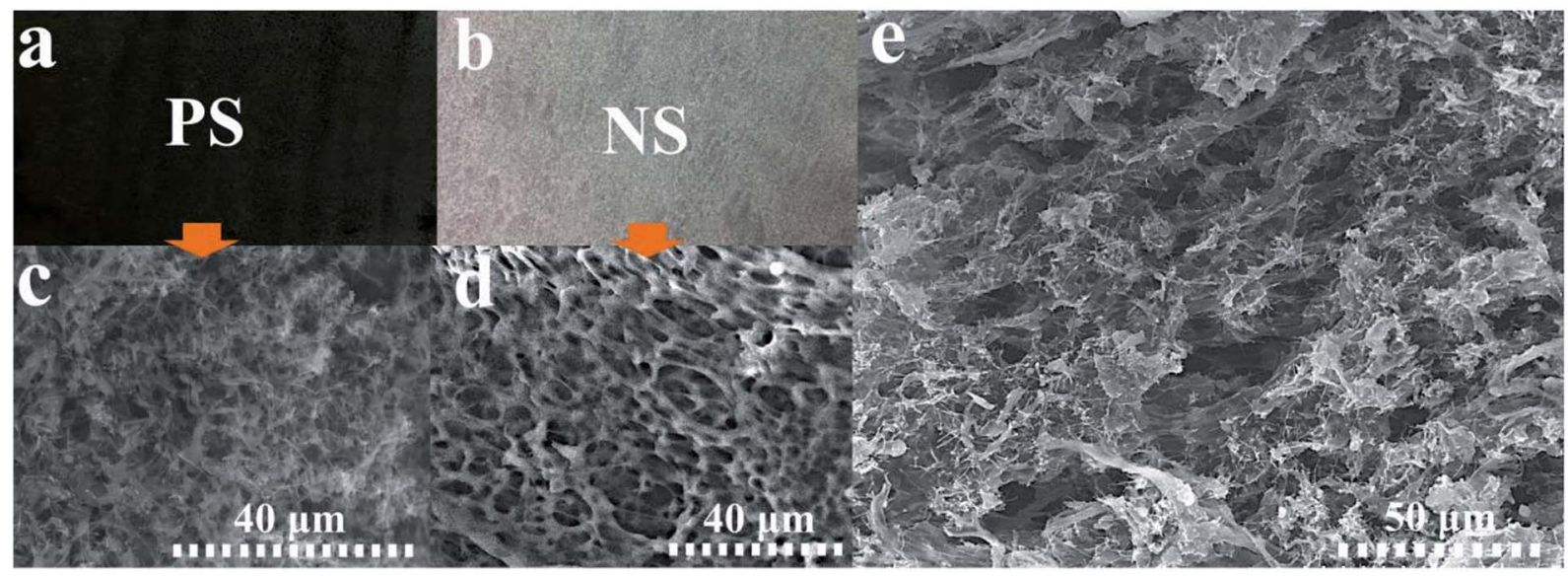

Fig. 3 (a-d) Digital images of positive and negative sides of gradient VGCF(aSMPUF and corresponding FESEM morphologies; (e) cross sectional view of gradient VGCFaSMPUF.
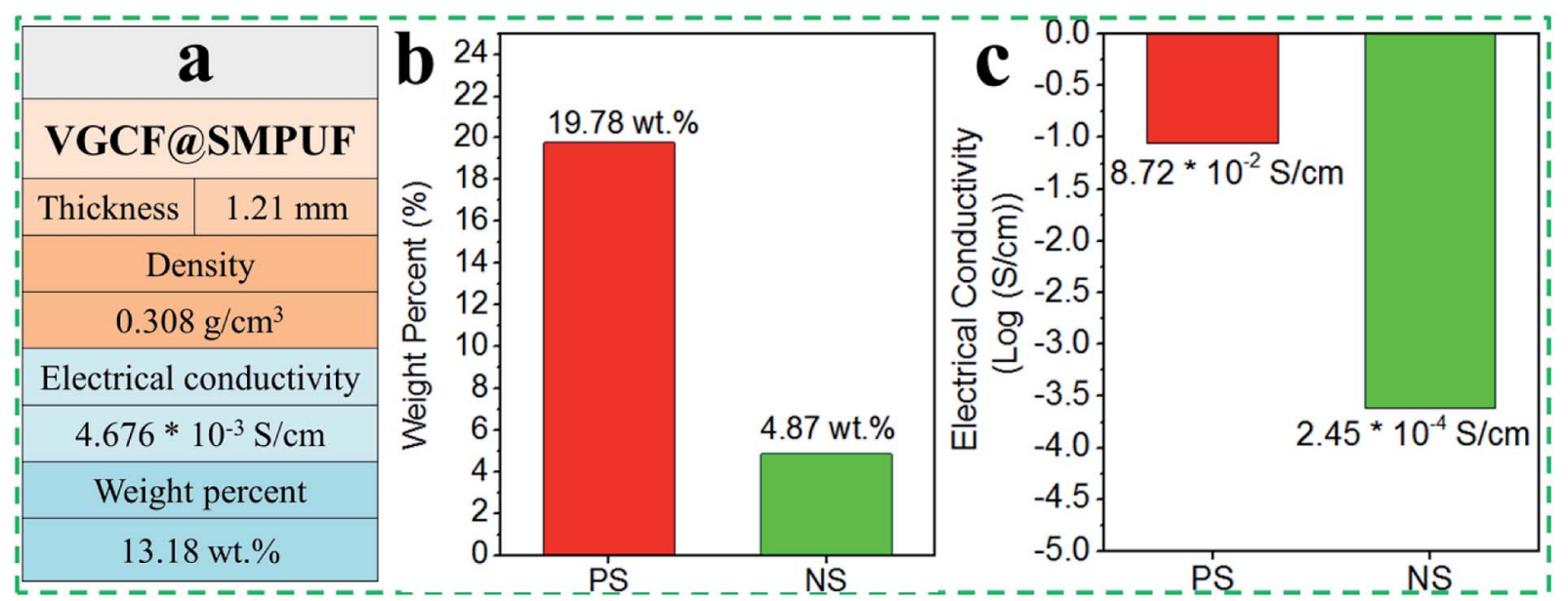

Fig. 4 (a) Structure information of VGCFaSMPUF; ( $b$ and c) weight contents and electrical conductivities for PS and NS of VGCFaSMPUF.

solution A was removed, and $10 \mathrm{~mL}$ of solution C (SMPU/DMF), having the same SMPU concentration as that of solution A, was added. The mixture was then sonicated for $5 \mathrm{~min}$ and magnetically stirred for $5 \mathrm{~min}$ in turn to uniformly disperse the VGCF. Next, the film formed on the PP substrate was suspended in air and dried slowly by fan at room temperature (RT) for 1 week. The film was peeled off and dried in an oven at $80{ }^{\circ} \mathrm{C}$ for 48 h. Finally, the obtained gradient VGCF@SMPUF was prepressed at $80^{\circ} \mathrm{C}$ for $12 \mathrm{~h}$. Pure SMPUF was similarly prepared by alternate dipping into an $80 \mathrm{mg} \mathrm{mL}^{-1} \mathrm{SMPU} / \mathrm{DMF}$ solution and DL water for 25 cycles.

\subsection{Characterization}

The morphologies and structures of the VGCF@SMPUF specimens were characterized separately by field emission scanning electron microscope (FE-SEM, Hitachi S-5000, $20 \mathrm{kV}$ ) and Raman spectrometer with $532 \mathrm{~nm}$ laser excitation (HoloLab series 5000, Kaiser Optical Systems). Conductivity was measured by MCP-HT450 conductivity meter (Dia Instruments Co.) using standard four-probe method, and mechanical

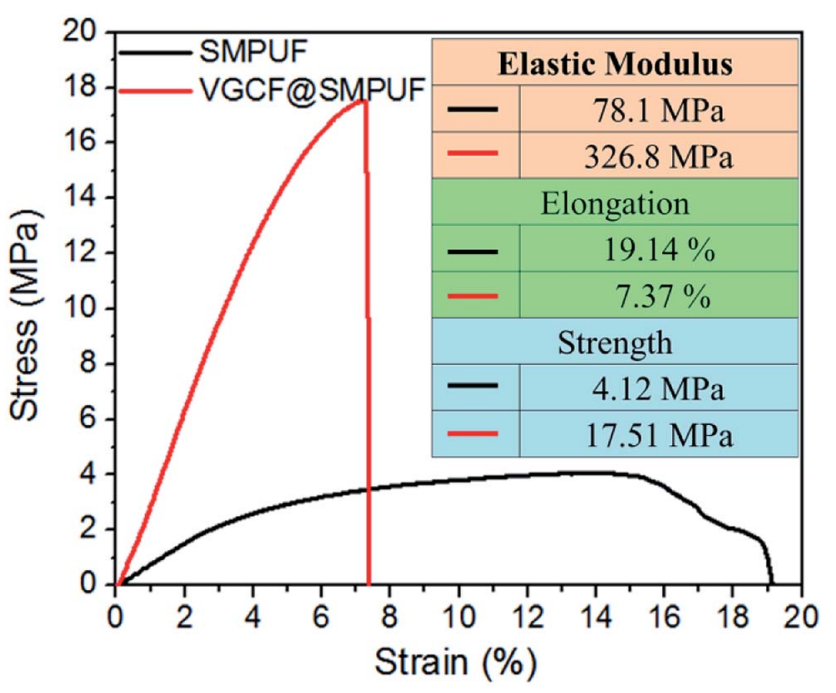

Fig. 5 Strain-stress curves of pure SMPUF, and gradient VGCF@SMPUF. 


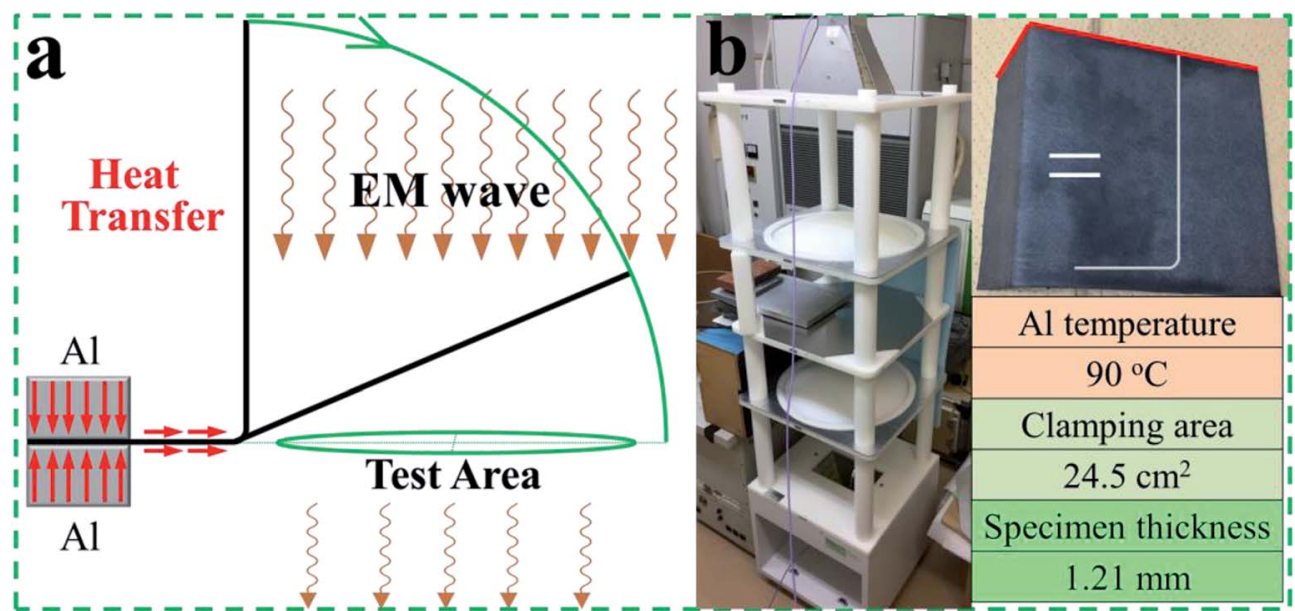

Fig. 6 (a) Measurement mechanism of electromagnetic shielding; (b) measurement devices, and conditions of electromagnetic shielding

properties were measured by Tensilon tensile tester (RTC1250A, A\&D Co.). The weight percentages of the VGCF composition at PS and NS were regarded as the VGCF contents of the VGCF@SMPU in the initial and final dipping solutions (VGCF@SMPU/DMF). These weight percentages were indirectly calculated by precipitating the dipping solution to obtain the weight of VGCF@SMPU, and re-dissolving and centrifuging the
SMPU composition of the dried VGCF@SMPU to obtain the weight of the VGCF.

The microwave shielding properties were evaluated in the frequency range between 6 and $16 \mathrm{GHz}$ with a transmission attenuation measurement system (KEYCOM Corp.) connected to a vector network analyzer $(37247 \mathrm{D}$, Anritsu Co. Ltd.) to calculate the shielding effectiveness (SE). A heating device was equipped near the circular test area $(\Phi 7.5 \mathrm{~cm})$ of the system to
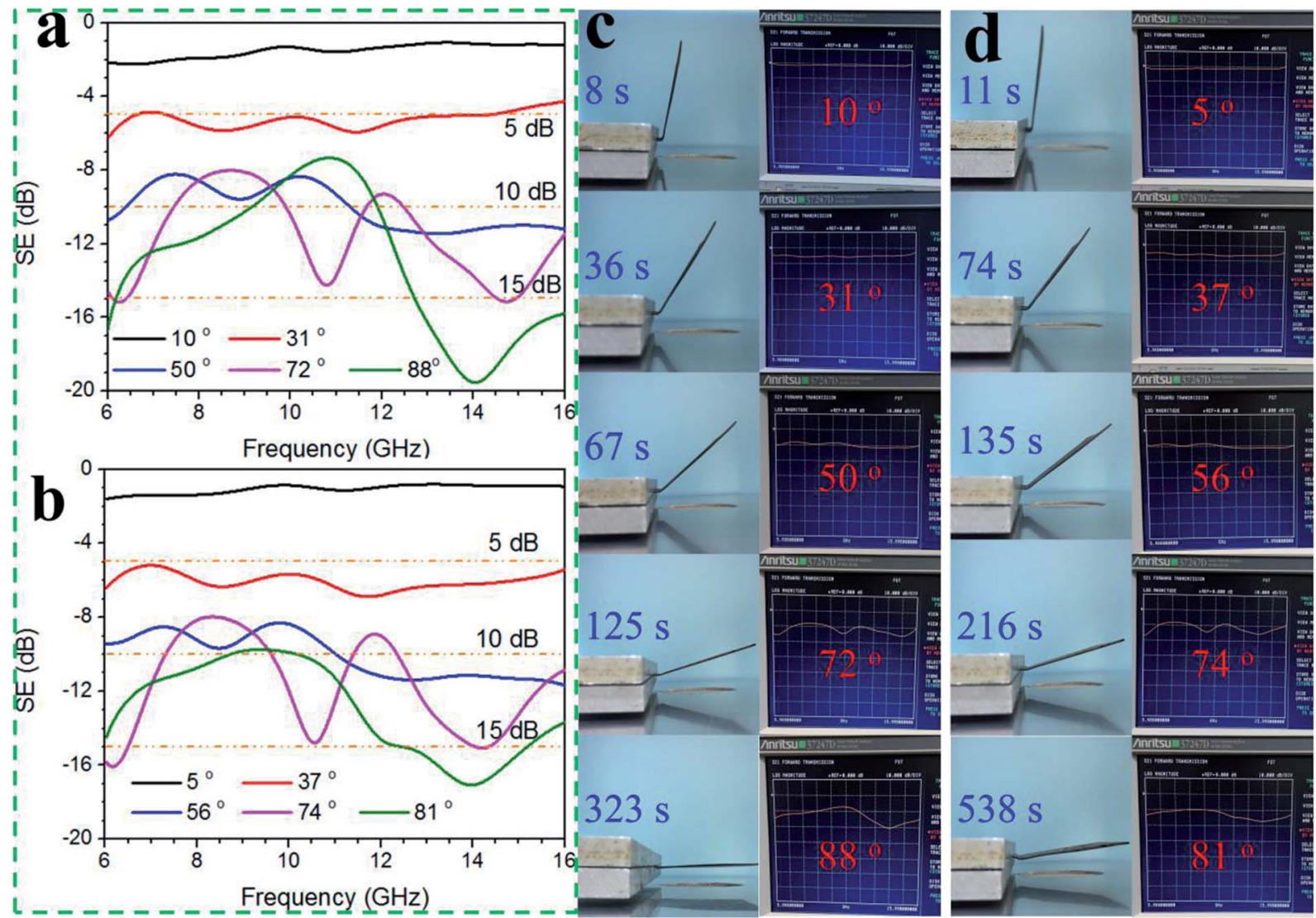

Fig. 7 SE change of (a) PS and (b) NS with increasing recovery angle; angle variation and corresponding SE value of (c) PS and (d) NS of gradient VGCFASMPUF. 
measure the variation of the shielding effectiveness during shape recovery. A VGCF@SMPUF specimen that was bent to the positive side (PS) (i.e., the higher VGCF content side) recovers from the PS, and is denoted as the recovery from the PS. The electromagnetic waves entered from this side. In a similar way, we describe the VGCF@SMPUF that was bent to the negative side (NS) (i.e., the lower VGCF content side) recovers from the NS.

\section{Results and discussion}

The VGCF had a diameter of approximately $150 \mathrm{~nm}$ and a length of a few micrometers, as shown in Fig. S1.† Raman measurement verified the presence of $D$ and G feature peaks of VGCF (Fig. 2). The SMPU used in this research, as previously studied in our group, is a typical shape memory polymer with a glass transition temperature $\left(T_{\mathrm{g}}\right)$ of $\sim 65^{\circ} \mathrm{C}$, and melting temperature $\left(T_{\mathrm{m}}\right)$ of $\sim 170{ }^{\circ} \mathrm{C} \cdot{ }^{\mathbf{4 1 , 4 2}}$ The polymer was obtained by polyaddition of linear chain diisocyanate and polyol (Fig. 1b). The amino $(-\mathrm{NH}-)$ and ketone (-CO-) groups in its structure, carbamate (-NH-CO-O-), were detected by FTIR, as shown in Fig. S2. $\dagger$

The as-obtained VGCF@SMPUF had a black positive side and a white negative side with a porous morphology, as shown in Fig. 3a-d. Compared with the low content of VGCF on the negative side, the higher content of VGCF on the positive side obviously impeded the formation of a continuous SMPU phase (Fig. 3c). For the formation of porous structure on the negative side, we attribute it to the slow evaporation of DL water during drying process (Fig. 3d). Owing to the strong solubility of DMF for the SMPUF component, inter-laminar fusion led to a subtle layer structure in the VGCF@SMPUF (Fig. 3e).

This gradient VGCF@SMPUF was $1.21 \mathrm{~mm}$ thick with a density of $\sim 0.31 \mathrm{~g} \mathrm{~cm}^{-3}$ and a VGCF content of $13.18 \mathrm{wt} \%$, which exhibited an electrical conductivity $(\sigma)$ of $\sim 4.68 \times$ $10^{-3} \mathrm{~S} \mathrm{~cm}^{-1}$ (Fig. 4a). By comparison the PS and NS showed weight contents and electrical conductivities of approximately $19.78 \mathrm{wt} \%$ and $4.87 \mathrm{wt} \%$, and $8.72 \times 10^{-2} \mathrm{~S} \mathrm{~cm}^{-1}$ and $2.45 \times$ $10^{-4} \mathrm{~S} \mathrm{~cm}^{-1}$, respectively (Fig. $4 \mathrm{~b}$ and c). This result suggested successful fabrication of a gradient VGCF@SMPUF. Strainstress curves indicated that the addition of VGCF into SMPUF enhanced the elastic modulus and mechanical strength of the VGCF@SMPUF to be approximately 4 times as high as their original values, whereas the elongation decreased to be more than 2 times as high as its original value (Fig. 5). We conclude that the addition of VGCF increased the strength and lowered the flexibility of this SMPUF. This result is consistent with most studies that carbon nanofiber or nanotube based fillers have mechanical reinforcement effect on polymer matrices ${ }^{43-46}$

The SMPU used in this research is a typical temperatureresponding one-way shape memory polymer. The shape recovery effect occurs when heating up to the $T_{\mathrm{g}}$ point of the polymer (approximately $65{ }^{\circ} \mathrm{C}$ ). The VGCF@SMPUF fabricated based on this shape memory polymer was used to investigate the shape memory driven microwave shielding performance. Variation of the SE with the angle recovery was evaluated by the electromagnetic coverage extent of the VGCF@SMPUF to the circular testing area (Fig. 6a and b). One side of the
VGCF@SMPUF was expanded from the initially fixed right-angle status. The other side was clamped by two aluminum (Al) plates pre-heated to $90{ }^{\circ} \mathrm{C}$. Thus, angle recovery of the VGCF@SMPUF bent to a right angle was achieved by heat transfer from the hot Al plates to VGCF@SMPUF.

As shown in Fig. 7a and b, the shielding result suggests that the SE of the VGCF@SMPUF increased as the recovery angle increased in the frequency range of 6-16 GHz. When the VGCF@SMPUF, which was initially bent to PS and NS, recovered from the PS and NS upon heat transfer, there was no obvious difference in the SE values. For the two cases, VGCF@SMPUF gave a similar $\mathrm{SE}$ at a similar recovery angle. However, the recovery time from the PS and NS were obviously different. As shown in Fig. 7c and d, recovery from the PS required $323 \mathrm{~s}$ whereas recovery from the NS required $538 \mathrm{~s}$. We attribute these results to the different mechanical strength of PS and NS of the VGCF@SMPUF. The mechanism is discussed in the following section.

VGCF@SMPUF specimens with thicknesses varying from 1.21 to $0.11 \mathrm{~mm}$ were obtained by hot compression. Microwave shielding evaluations indicated an increase of the SE value with decreasing thickness. As shown in Fig. 8a and b, the gradient of the $0.11 \mathrm{~mm}$ thick VGCF@SMPUF sample reached a SE value up

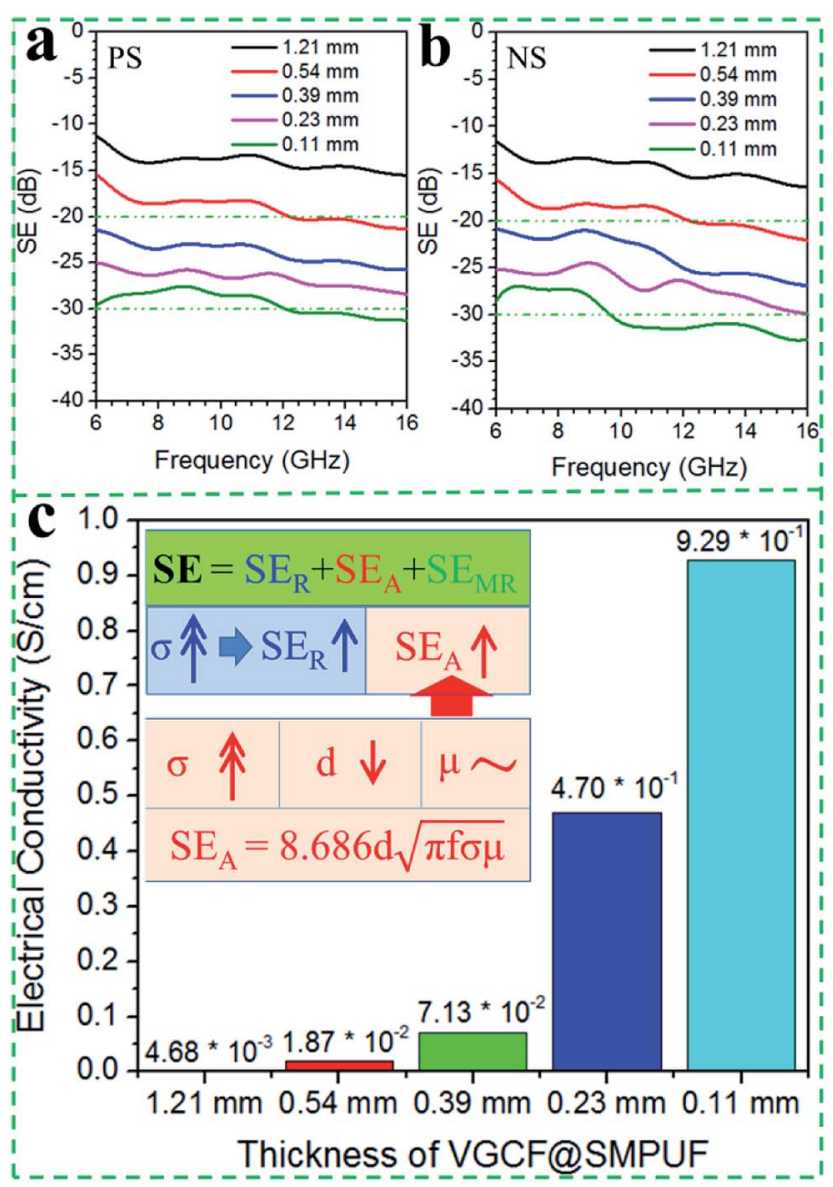

Fig. 8 Shielding effectiveness of (a) PS and (b) NS of the VGCF@SMPUF specimens at different extents of compression; (c) electrical conductivities, and change of $S_{A}$ and $S E_{R}$ for the VGCF@SMPUF specimens with different thickness compression. 


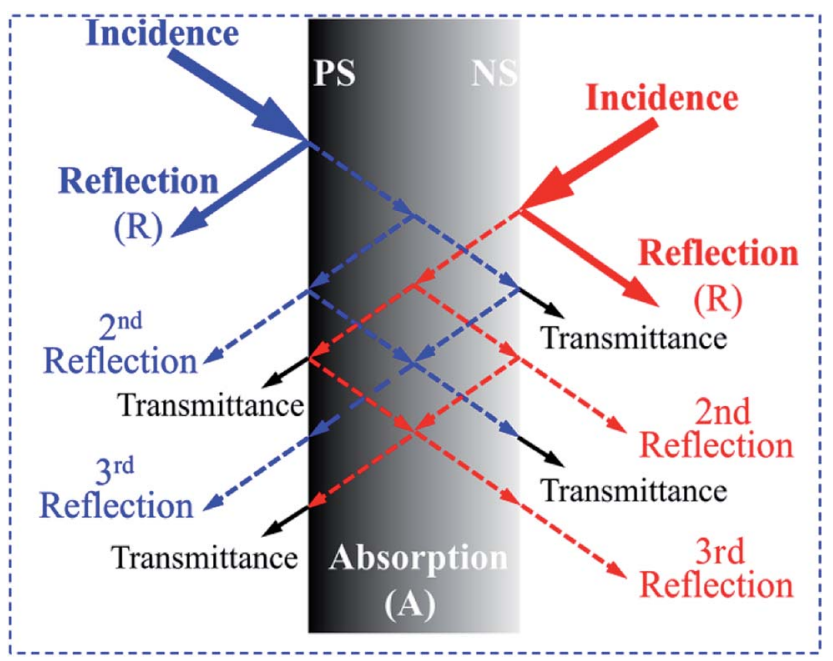

Fig. 9 Reflection effect from the PS and NS of gradient VGCFASMPUF.

to $\sim 30 \mathrm{~dB}$ (i.e., a shielding efficiency of $99.9 \%$ ) in the frequency range of 6-16 GHz. As for the reason, the shielding effectiveness is the sum of the reflection $\left(\mathrm{SE}_{\mathrm{R}}\right)$, absorption $\left(\mathrm{SE}_{\mathrm{A}}\right)$, and multiple reflection $\left(\mathrm{SE}_{\mathrm{MR}}\right)$. Improved electrical conductivity $(\sigma)$ generally results in an increase of $\mathrm{SE}_{\mathrm{R}}$. Therefore, the considerable increase of $\sigma$, which was attributed to continuous compression, increased $\mathrm{SE}_{\mathrm{R}}$. As shown in Fig. 8c, the VGCF@SMPUF at a thickness of $0.11 \mathrm{~mm}$ had $\sigma$ values more than $\sim 200$ times as great as that of original VGCF@SMPUF with a $1.21 \mathrm{~mm}$ thickness. Additionally, due to non-magnetic properties of the VGCF@SMPUF, compression is considered to have no obvious effect on permeability $(\mu)$, as shown in the inset of Fig. 8c. Thus, the value of $\mathrm{SE}_{\mathrm{A}}$ increased despite the decrease of thickness. Given that the part of $\mathrm{SE}_{\mathrm{MR}}$ can be neglected here (>15 dB), as a result, the total SE increased with compression. This indicates that the increase in $\mathrm{SE}$ is attributed to an increase of both reflection and absorption.

A comparison of the shielding properties indicates that the PS and NS of the VGCF@SMPUF specimens with different thickness had similar shielding effects in the frequency range of $6-16 \mathrm{GHz}$, as shown in Fig. 8a and b. In fact, the $\mathrm{SE}_{\mathrm{R}}$ parts of those VGCF@SMPUF specimens were considered to be approximately the same because there should be no obvious change of $\mathrm{SE}_{\mathrm{A}}$ when the microwave entered from either side of the VGCF@SMPUF. This result suggests that for a nonhomogeneous shielding composite, $\mathrm{SE}_{\mathrm{R}}$ not only depends on the surface properties, such as surface resistance and permittivity, but also relies on internal properties of the shielding composite. In other words, $\mathrm{SE}_{\mathrm{R}}$ is the sum of the surface and internal reflection, thus, making the $\mathrm{SE}_{\mathrm{R}}$ of PS and NS similar, as illustrated in Fig. 9.

Then, we investigated the recovery time and ratio, and fixity ratio of this gradient VGCF@SMPUF at different extents of compression. As shown in Fig. 10a-c, hot compression induced a faster recovery of the VGCF@SMPUF and a higher recovery and fixity ratio. The VGCF@SMPUF at thicknesses of 0.23 and $0.11 \mathrm{~mm}$ recovered to $100 \%$ within tens of seconds. We attribute this result to compaction of the VGCF component, which thus improved the thermal conductivity of the gradient VGCF@SMPUF. As detected from its bending point, when clamped by the Al plates, the VGCF@SMPUF compressed to a thickness of $0.11 \mathrm{~mm}$ showed a faster elevation temperature than that of the sample that had not been subjected hot-compression (Fig. 11).

In addition, the VGCF@SMPUF that was bent to the PS, showed a faster recovery time and higher recovery ratio than that bent to the NS. As for the reason, recovery of bending is a process where stretching (inner edge) and compression (outer edge) at the bending point proceeds simultaneously. The outer edge of this gradient VGCF@SMPUF, bent to the NS, and had a greater mechanical compression strength than that bent to the PS owing to the higher VGCF content at the outer edge, as illustrated in Fig. 12a. Therefore, recovery from the PS was easier than from the NS. Notably, the recovery time, ratios, and bending point temperatures of this gradient VGCF@SMPUF became closer as hot-compression was increased, as shown in Fig. 10a, b and 11. We attribute this result to the approaching VGCF contents and properties of the two sides with

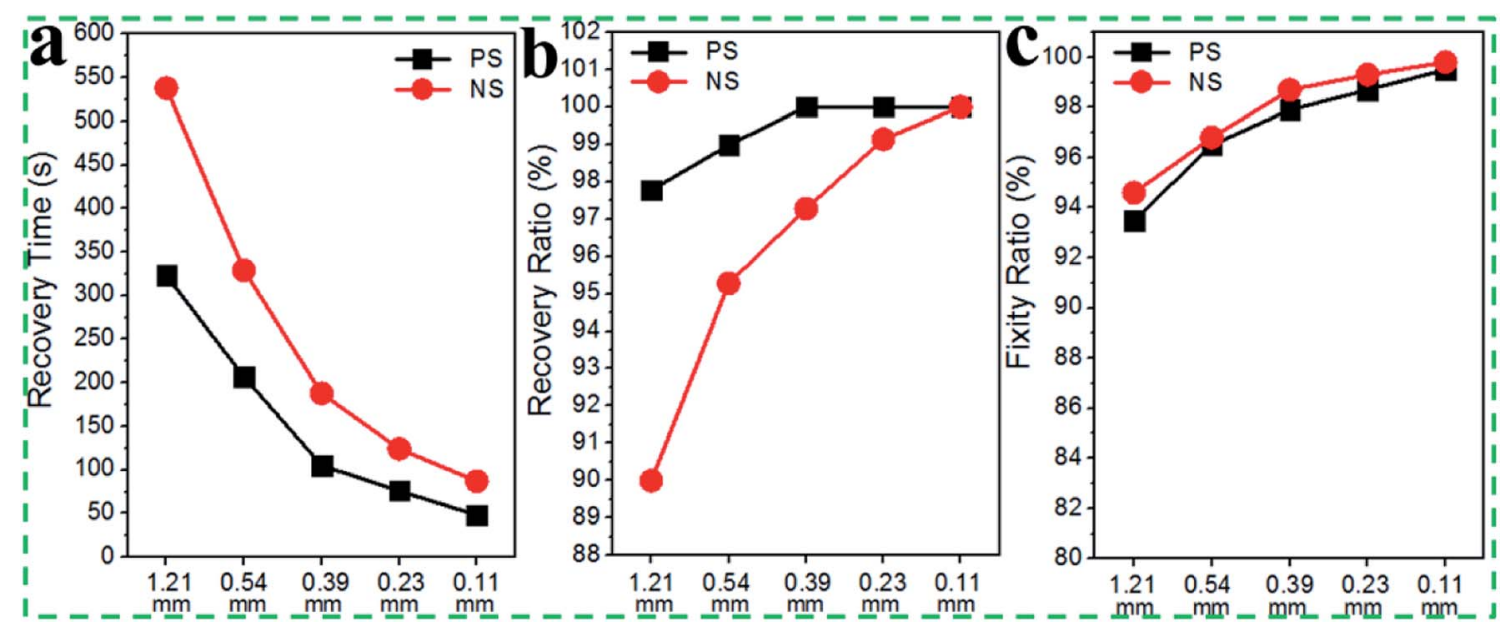

Fig. 10 (a) Recovery time, (b) recovery ratio, and (c) fixity ratio of PS and NS of the VGCF(aSMPUF specimens with different compression, which were initially bent and fixed at a right angle. 


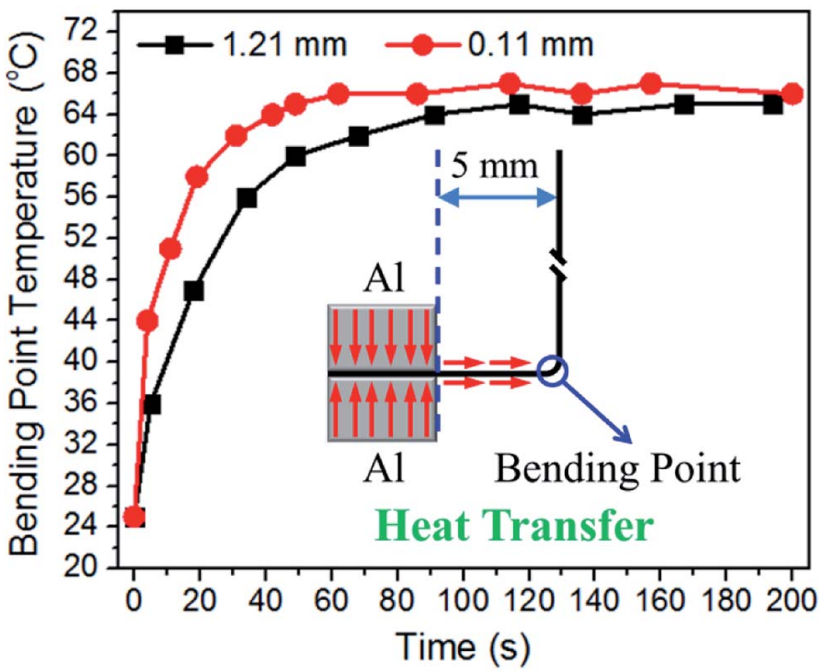

Fig. 11 Temperature change of the bending point with time for VGCF@SMPUF specimens with thicknesses of 1.21 and $0.11 \mathrm{~mm}$.

continuous compression (Fig. 12b). Therefore, we conclude that this gradient fabrication of VGCF content featured different shape recovery efficiencies in the two sides (PS and

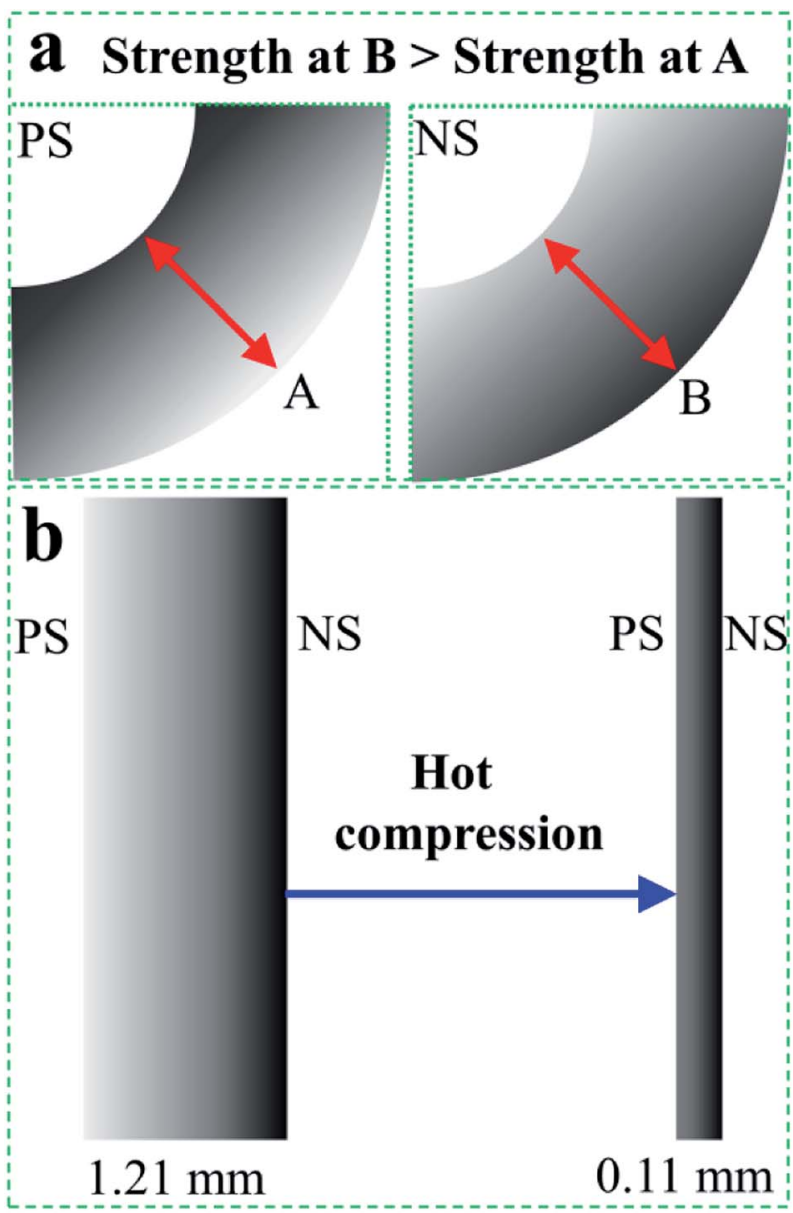

Fig. 12 (a) Comparison of the compression strength at the outer edges of PS and NS of gradient VGCF(aSMPUF; (b) approaching VGCF contents of PS and NS with continuous hot compression.
NS). This VGCF@SMPUF may have potential applications in the field of structural transformations of functional shielding devices.

This gradient VGCF@SMPUF functionalized traditional EM shielding material, and achieved the adjustment of EM shielding performance through the angle recovery. The shape memory effect was achieved by heat transfer of thermally conductive VGCF. The shielding effectiveness of this VGCF@SMPUF was controlled by different degrees of shape recovery. Moreover, the gradient distribution of the VGCF could directionally improve the efficiency of the angle recovery, such as the recovery ratio and time. However, there are also some limitations to this VGCF@SMPUF. For examples, thick VGCF@SMPUF has decreased angle fixity and recover ratio, which affect the precision of control and adjustment of VGCF@SMPUF devices. This aspect might be improved by adjusting the mechanical strength of the two sides, which can be achieved by changing the gradient content of the VGCF in the thickness direction. Recently, some approaches based on three-point bending have been developed in order to measure bending recovery effect. ${ }^{47-49}$ Therefore, we are intended to quantitatively investigate the bending recovery force and ratio of this gradient VGCF@SMPUF by referring those researches. In addition, the shape recovery of this gradient VGCF@SMPUF is also irreversible, which decreases the practicality and novelty of the device. In the following research, we may consider the development of reversible VGCF@SMP (i.e., two-way SMPs).

\section{Conclusion}

Gradient vapor grown carbon fiber-based shape memory polyurethane foam (VGCF@SMPUF) was fabricated by alternative dipping in a VGCF containing SMPU/DMF solution of decreasing concentration and distilled water to form shape memory driven adjustable microwave shielding. Different electrical conductivities and VGCF contents on two sides of this gradient VGCF@SMPUF were obtained. The adjustability of the shielding effectiveness was achieved by different degrees of angle recovery. Hot compression could not only decrease the recovery time, and gave a higher recovery ratio, but also clearly improve the shielding performance of this gradient VGCF@SMPUF. Moreover, the VGCF@SMPUF recovering from the side with a higher VGCF content exhibited a faster recovery time and higher recovery ratio than that recovering from the side with a lower VGCF content owing to different mechanical strengths at either side.

\section{Conflicts of interest}

There are no conflicts to declare.

\section{Acknowledgements}

This work was supported by JSPS (MEXT financial supports) KAKENHI No. $15 \mathrm{H} 01789$ and 26420721. We also express our thanks to administrative staff of Shinshu University Sano 
Sumika for her lots of assistance on English modification and article processing for this paper.

\section{References}

1 Z. Chen, C. Xu, C. Ma, W. Ren and H. M. Cheng, Lightweight and flexible graphene foam composites for highperformance electromagnetic interference shielding, $A d v$. Mater., 2013, 25(9), 1296-1300.

2 N. Yousefi, X. Sun, X. Lin, X. Shen, J. Jia, B. Zhang and J. K. Kim, Highly aligned graphene/polymer nanocomposites with excellent dielectric properties for high-performance electromagnetic interference shielding, Adv. Mater., 2014, 26(31), 5480-5487.

3 Y. Wu, Z. Wang, X. Liu, X. Shen, Q. Zheng, Q. Xue and J. K. Kim, Ultralight graphene foam/conductive polymer composites for exceptional electromagnetic interference shielding, ACS Appl. Mater. Interfaces, 2017, 9(10), 90599069.

4 K. Zhang, H. O. Yu, Y. D. Shi, Y. F. Chen, J. B. Zeng, J. Guo and $\mathrm{M}$. Wang, Morphological regulation improved electrical conductivity and electromagnetic interference shielding in poly (L-lactide)/poly ( $\varepsilon$-caprolactone)/carbon nanotube nanocomposites via constructing stereocomplex crystallites, J. Mater. Chem. C, 2017, 5(11), 2807-2817.

5 Z. Zeng, H. Jin, M. Chen, W. Li, L. Zhou, X. Xue and Z. Zhang, Microstructure design of lightweight, flexible, and high electromagnetic shielding porous multiwalled carbon nanotube/polymer composites, Small, 2017, 13(34), 1701388.

6 K. Zhang, G. H. Li, L. M. Feng, N. Wang, J. Guo, K. Sun and M. Wang, Ultralow percolation threshold and enhanced electromagnetic interference shielding in poly (L-lactide)/ multi-walled carbon nanotube nanocomposites with electrically conductive segregated networks, J. Mater. Chem. C, 2017, 5(36), 9359-9369.

7 M. Rahaman, T. K. Chaki and D. Khastgir, Development of high performance EMI shielding material from EVA, NBR, and their blends: effect of carbon black structure, J. Mater. Sci., 2011, 46(11), 3989-3999.

8 Y. Yan, H. Xia, Y. Qiu, Z. Xu and Q. Q. Ni, Shape memory driving thickness-adjustable G@SMPU sponge with ultrahigh carbon loading ratio for excellent microwave shielding performance, Mater. Lett., 2019, 236, 116-119.

9 W. L. Song, J. Wang, L. Z. Fan, Y. Li, C. Y. Wang and M. S. Cao, Interfacial engineering of carbon nanofibergraphene-carbon nanofiber heterojunctions in flexible lightweight electromagnetic shielding networks, ACS Appl. Mater. Interfaces, 2014, 6(13), 10516-10523.

10 S. Sankaran, K. Deshmukh, M. B. Ahamed and S. K. Pasha, Recent advances in electromagnetic interference shielding properties of metal and carbon filler reinforced flexible polymer composites: a review, Composites, Part A, 2018, 114, 49-71.

11 M. H. Al-Saleh, W. H. Saadeh and U. Sundararaj, EMI shielding effectiveness of carbon based nanostructured polymeric materials: a comparative study, Carbon, 2013, 60, 146-156.
12 W. L. Song, M. S. Cao, M. M. Lu, S. Bi, C. Y. Wang, J. Liu and L. Z. Fan, Flexible graphene/polymer composite films in sandwich structures for effective electromagnetic interference shielding, Carbon, 2014, 66, 67-76.

13 J. Ling, W. Zhai, W. Feng, B. Shen, J. Zhang and W. G. Zheng, Facile preparation of lightweight microcellular polyetherimide/graphene composite foams for electromagnetic interference shielding, ACS Appl. Mater. Interfaces, 2013, 5(7), 2677-2684.

14 X. Hong and D. D. L. Chung, Carbon nanofiber mats for electromagnetic interference shielding, Carbon, 2017, 111, 529-537.

15 W. L. Song, X. T. Guan, L. Z. Fan, W. Q. Cao, C. Y. Wang and M. S. Cao, Tuning three-dimensional textures with graphene aerogels for ultra-light flexible graphene/texture composites of effective electromagnetic shielding, Carbon, 2015, 93, 151-160.

16 L. Zou, S. Zhang, X. Li, C. Lan, Y. Qiu and Y. Ma, Step-by-Step Strategy for Constructing Multilayer Structured Coatings Toward High-Efficiency Electromagnetic Interference Shielding, Adv. Mater. Interfaces, 2016, 3(5), 1500476.

17 X. Cai, M. Hu, D. Zhang, G. Hu and J. Yang, Experimental Study on Tunable Electromagnetic Shielding by Microlattice Materials with Organized Microstructures, Adv. Eng. Mater., 2018, 1700823.

18 L. Q. Zhang, B. Yang, J. Teng, J. Lei, D. X. Yan, G. J. Zhong and Z. M. Li, Tunable electromagnetic interference shielding effectiveness via multilayer assembly of regenerated cellulose as a supporting substrate and carbon nanotubes/polymer as a functional layer, J. Mater. Chem. C, 2017, 5(12), 3130-3138.

19 M. Mishra, A. P. Singh, V. Gupta, A. Chandra and S. K. Dhawan, Tunable EMI shielding effectiveness using new exotic carbon: polymer composites, J. Alloys Compd., 2016, 688, 399-403.

20 L. Lu, D. Xing, K. S. Teh, H. Liu, Y. Xie, X. Liu and Y. Tang, Structural effects in a composite nonwoven fabric on EMI shielding, Mater. Des., 2017, 120, 354-362.

21 Y. Li, B. Shen, D. Yi, L. Zhang, W. Zhai, X. Wei and W. Zheng, The influence of gradient and sandwich configurations on the electromagnetic interference shielding performance of multilayered thermoplastic polyurethane/graphene composite foams, Compos. Sci. Technol., 2017, 138, 209-216.

22 B. Zhao and C. B. Park, Tunable electromagnetic shielding properties of conductive poly (vinylidene fluoride)/Ni chain composite films with negative permittivity, J. Mater. Chem. C, 2017, 5(28), 6954-6961.

23 S. H. Lee, S. Yu, F. Shahzad, W. N. Kim, C. Park, S. M. Hong and C. M. Koo, Density-tunable lightweight polymer composites with dual-functional ability of efficient EMI shielding and heat dissipation, Nanoscale, 2017, 9(36), 13432-13440.

24 B. Shen, Y. Li, D. Yi, W. Zhai, X. Wei and W. Zheng, Strong flexible polymer/graphene composite films with 3D sawtooth folding for enhanced and tunable electromagnetic shielding, Carbon, 2017, 113, 55-62. 
25 Y. Wang, X. D. Cheng, W. L. Song, C. J. Ma, X. M. Bian and M. Chen, Hydro-sensitive sandwich structures for selftunable smart electromagnetic shielding, Chem. Eng. J., 2018, 344, 342-352.

26 Z. Chen, D. Yi, B. Shen, L. Zhang, X. Ma, Y. Pang and W. Zheng, Semi-transparent biomass-derived macroscopic carbon grids for efficient and tunable electromagnetic shielding, Carbon, 2018, 139, 271-278.

27 Y. Dong, Y. Zhu, Y. Zhao, F. Liu, E. Wang and Y. Fu, Enhance interfacial properties of glass fiber/epoxy composites with environment-friendly water-based hybrid sizing agent, Composites, Part A, 2017, 102, 357-367.

$28 \mathrm{~J}$. Hu, Y. Zhu, H. Huang and J. Lu, Recent advances in shapememory polymers: Structure, mechanism, functionality, modeling and applications, Prog. Polym. Sci., 2012, 37(12), 1720-1763.

29 Q. Zhao, H. J. Qi and T. Xie, Recent progress in shape memory polymer: new behavior, enabling materials, and mechanistic understanding, Prog. Polym. Sci., 2015, 49, 79120.

30 E. Wang, Y. Dong, M. Z. Islam, L. Yu, F. Liu, S. Chen and $\mathrm{N}$. Hu, Effect of graphene oxide-carbon nanotube hybrid filler on the mechanical property and thermal response speed of shape memory epoxy composites, Compos. Sci. Technol., 2019, 169, 209-216.

31 M. D. Hager, S. Bode, C. Weber and U. S. Schubert, Shape memory polymers: past, present and future developments, Prog. Polym. Sci., 2015, 49, 3-33.

32 L. Sun, W. M. Huang, Z. Ding, Y. Zhao, C. C. Wang, H. Purnawali and C. Tang, Stimulus-responsive shape memory materials: a review, Mater. Des., 2012, 33, 577-640.

33 Y. Yao, T. Zhou, Y. Xu, Y. Liu and J. Leng, Preparation and characterization of shape memory composite foams based on solid foaming method, J. Appl. Polym. Sci., 2018, 135(46), 46767.

34 B. Jin, H. Song, R. Jiang, J. Song, Q. Zhao and T. Xie, Programming a crystalline shape memory polymer network with thermo-and photo-reversible bonds toward a singlecomponent soft robot, Sci. Adv., 2018, 4(1), eaao3865.

35 Y. Yan, H. Xia, Y. Qiu, Z. Xu and Q. Q. Ni, Multi-layer graphene oxide coated shape memory polyurethane for adjustable smart switches, Compos. Sci. Technol., 2019, 172, 108-116.

36 Y. Huang, M. Zhu, Z. Pei, Q. Xue, Y. Huang and C. Zhi, A shape memory supercapacitor and its application in smart energy storage textiles, J. Mater. Chem. A, 2016, 4(4), 12901297.

37 M. Zarek, N. Mansour, S. Shapira and D. Cohn, 4D Printing of Shape Memory-Based Personalized Endoluminal Medical Devices, Macromol. Rapid Commun., 2017, 38(2), 1600628.
38 Y. S. Wong, A. V. Salvekar, K. Da Zhuang, H. Liu, W. R. Birch, K. H. Tay and S. S. Venkatraman, Bioabsorbable radiopaque water-responsive shape memory embolization plug for temporary vascular occlusion, Biomaterials, 2016, 102, 98106.

39 W. Zhao, L. Liu, X. Lan, B. Su, J. Leng and Y. Liu, Adaptive repair device concept with shape memory polymer, Smart Mater. Struct., 2017, 26(2), 025027.

40 H. Wei, Q. Zhang, Y. Yao, L. Liu, Y. Liu and J. Leng, Directwrite fabrication of $4 \mathrm{D}$ active shape-changing structures based on a shape memory polymer and its nanocomposite, ACS Appl. Mater. Interfaces, 2016, 9(1), 876-883.

41 H. Chen, H. Xia, Y. Qiu and Q. Q. Ni, Analyzing effects of interfaces on recovery rates of shape memory composites from the perspective of molecular motions, Compos. Sci. Technol., 2018, 163, 105-115.

$42 \mathrm{H}$. Chen, H. Xia and Q. Q. Ni, Study on material performances of lead zirconate titanate/shape memory polyurethane composites combining shape memory and piezoelectric effect, Composites, Part A, 2018, 110, 183-189.

43 Y. K. Choi, K. I. Sugimoto, S. M. Song, Y. Gotoh, Y. Ohkoshi and $\mathrm{M}$. Endo, Mechanical and physical properties of epoxy composites reinforced by vapor grown carbon nanofibers, Carbon, 2005, 43(10), 2199-2208.

44 M. H. Al-Saleh and U. Sundararaj, Review of the mechanical properties of carbon nanofiber/polymer composites, Composites, Part A, 2011, 42(12), 2126-2142.

45 A. Baji, Y. W. Mai, S. C. Wong, M. Abtahi and X. Du, Mechanical behavior of self-assembled carbon nanotube reinforced nylon 6, 6 fibers, Compos. Sci. Technol., 2010, 70(9), 1401-1409.

46 S. Rahmanian, A. R. Suraya, M. A. Shazed, R. Zahari and E. S. Zainudin, Mechanical characterization of epoxy composite with multiscale reinforcements: carbon nanotubes and short carbon fibers, Mater. Des., 2014, 60, 34-40.

47 F. Li, F. Scarpa, X. Lan, L. Liu, Y. Liu and J. Leng, Bending shape recovery of unidirectional carbon fiber reinforced epoxy-based shape memory polymer composites, Composites, Part A, 2019, 116, 169-179.

48 M. Fejős, G. Romhány and J. Karger-Kocsis, Shape memory characteristics of woven glass fibre fabric reinforced epoxy composite in flexure, J. Reinf. Plast. Compos., 2012, 31(22), 1532-1537.

49 M. Fejős, Shape memory performance of asymmetrically reinforced epoxy/carbon fibre fabric composites in flexure, eXPRESS Polym. Lett., 2013, 7(6), 528-534. 